\title{
Keynote Speech: Underexplored Research Topics from the Commercial World
}

\author{
Erik Hoel \\ Redlands, CA, USA \\ ehoel@esri.com
}

\begin{abstract}
Active research in the spatio-temporal database domain is approaching fifty years of age, beginning with the early research by Waldo Tobler at Michigan, Roger Tomlinson on CGIS, Edgar Horwood at Washington, Howard Fisher at Harvard, and Donald Cooke at Census. Very significant progress has been made during this period, with spatial data now becoming ubiquitous with the current generation of web applications, imbedded mapping, smartphones, and location-based services. However, many of the most challenging problems being faced by industry in the spatio-temporal domain remain relatively unaddressed by the research community. Many of these problems are related to the development of technology and applications primarily intended for the defense and intelligence worlds. This domain generally involves:
\end{abstract}

- Prohibitively large quantities of data,

- Real time data fusion,

- Remote sensing (including video and multispectral),

- Large-scale automated and semi-automated feature extraction, and

- Geostreaming, including real-time/continuous analysis and geoprocessing.

In addition, in order to achieve scalability and elasticity, non-traditional architectures and data storage technologies (e.g., NoSQL and multi-tenant) are frequently employed. The guiding mantras of "simple scales, complex fails", as well as "precompute as if your life depends upon it" are key to success in these domains.

Finally, another area that deserves the enhanced attention of the research community involves complex interactions between vast collections of objects in time and space (e.g., migrations, flocking behavior, or communications), with the goal being to infer something about the processes going on. The spatial interaction domain is of particular significance to both social networking as well as intelligence. This talk will provide researchers with a discussion of these topics, presenting additional background and context to these difficult real-world problems along with an overview of what is currently considered the state of the art in framework architectures and production systems. 